AN. MED. INTERNA (Madrid) Vol. 19, N. $^{\circ} 9$, pp. $453-456,2002$

\title{
Prevalencia de obesidad en pacientes médicos hospitalizados
}

\author{
F. J. CARRASCO SÁNCHEZ, F. DÍAZ ALCAIDE, Y. MARÍN FERNÁNDEZ, I. \\ CHAPARRO MORENO, E. PUJOL DE LA LLAVE
}

Servicio de Medicina Interna. Hospital Juan Ramón Jiménez. Ronda Norte. Huelva

\author{
PREVALENCE OF OBESITY IN AN INTERNAL MEDICINE \\ DEPARTMENT
}

\section{RESUMEN}

Fundamento: Se describe la distribución ponderal de una muestra de pacientes médicos hospitalizados. Se estima la prevalencia global y su presencia entre diferentes variables clínicas.

Material y métodos: Se diseñó un estudio descriptivo de prevalencia y se determinó el peso, la talla y diversas variables clínicas de 101 pacientes hospitalizados en el Servicio de Medicina Interna del Hospital Juan Ramón Jiménez de Huelva. Los pacientes fueron ingresados entre los días 6-7 de junio de 2000. La obesidad fue definida por un índice de masa corporal (IMC) $\geq 30 \mathrm{Kg} / \mathrm{m}^{2}$.

Resultados: La prevalencia de la obesidad global fue de $32,7 \%$ $[0,236-0,416]$. En el estudio encontramos asociación estadísticamente significativa con el sexo femenino (Razón de prevalencia -RP- 3,22), HTA (RP 4,72), dislipemia (RP 4,40) e hiperuricemia (RP4,28).

Conclusiones: La prevalencia de obesidad de nuestros pacientes oscila entre el 23 y $41 \%$, por encima de las estimaciones sobre población general. Más prevalente entre las mujeres y asociada a los factores de riesgo cardiovasculares clásicos.

PALABRAS CLAVE: Prevalencia. Obesidad. Hospitalización. Medicina Interna.

\begin{abstract}
Objective: We describe the weight's distribution in a sample of medical patients in hospital. We estimate the global prevalence and the presence between other different clinical variables.

Material and Methods: A prevalence cross-sectional study was carried out. We determine weight, stature and several clinical variables in 101 patients admit in the internal medicine department of Juan Ramón Jiménez hospital in Huelva. The patients were admitted from 6th to 7 th of june in 2000. The Body Mass Index (BMI) $\geq 30 \mathrm{Kg} / \mathrm{m}^{2}$ was used to define the obesity.

Results: The prevalence of obesity was $32.2 \%$ [0.236 - 0.416]. In the study we find an association with female (prevalente rate $-P R-3,22$ ), HTA (PR 4.72), dislipemia (RP 4.40) and hyperuricacemia (RP 4.28).

Conclusions: The prevalence of obesity in our patients was between $23-41 \%$, it was greater than others estimations in general people. We find association with women and classic cardiovascular risk factors.
\end{abstract}

KEY WORDS: Prevalence. Obesity. Internal Medicine department.

Carrasco Sánchez FJ, Díaz Alcaide F, Marín Fernández Y, Chaparro Moreno I, Pujol de la Llave E. Prevalencia de obesidad en pacientes médicos hospitalizados. An Med Interna (Madrid) 2002; 19: 453-456.

\section{INTRODUCCIÓN}

La obesidad es un modelo de enfermedad sistémica de importancia relevante debido a su creciente prevalencia y a la comorbilidad que genera en otras entidades nosológicas (1). En la práctica clínica diaria los médicos se enfrentan a este problema de salud pública que condiciona el manejo de sus pacientes.

Se conocen en la literatura española numerosos estudios para estimar la prevalencia de la población general (2-6). Sin embargo, datos sobre población enferma son más escasos. En este sentido, el conocimiento de la magnitud de la obesidad de nuestros pacientes es un arma fundamental para el adecuado manejo clínicos de estos.

Existen numerosos métodos para la evaluación de la grasa corporal. La antropometría es el método empleado con mayor frecuencia tanto en la práctica clínica como en la investigación epidemiológica por ser sencillo y económico (7). El consenso español de 1995 para la evaluación de la obesidad elaborado por la Sociedad Española para el estudio de la Obesidad (SEEDO) recomiendan el empleo de la antropometría. El índice de masa corporal -IMC- o índice de Quetelet

Trabajo aceptado: 23 de mayo de 2002

Correspondencia: Dr FJ Carrasco Sánchez. Urbanización Aljapark. c/Rosa nº 8 (423). 21110 Aljaraque. Huelva. Teléfono: 959318539. Correo electrónico: fjcarrascos@wanadoo.es 
(peso en $\mathrm{Kg} /$ talla en $\mathrm{m}^{2}$ ) es el método más ampliamente validado y utilizado en los estudios epidemiológicos. Por su sencillez esta incorporado a la práctica clínica diaria (8).

Se pretende conocer la prevalencia de sobrepeso y obesidad en los pacientes que ingresan en el Servicio de Medicina Interna del Hospital Juan Ramón Jiménez de Huelva.

\section{MATERIAL Y MÉTODOS}

Se estudiaron la totalidad de los pacientes ingresados en el Servicio de Medicina Interna del Hospital Juan Ramón Jiménez de Huelva, incluyendo las secciones de neumología, neurología, medicina interna, digestivo, cardiología y nefrología durante un periodo de 48 horas comprendidas en los días 6 y 7 de Junio de 2000. Se diseñó un estudio descriptivo de prevalencia (cross-sectional), y un protocolo expresamente diseñado al efecto para recoger los datos de las variables antropométricas, clínicas y biológicas. El trabajo de campo fue realizado por 2 parejas de entrevistadores previamente adiestradas y habiendo consensuado los métodos de medidas.

La recogida de los datos clínicos y biológicos se lleva a cabo a partir de la historia clínica de cada enfermo, y de la información facilitada por el médico y la enfermera responsable del mismo. Las variables estudiadas fueron la edad, género, tabaquismo, hipertensión arterial, diabetes mellitus, dislipemia, sospecha clínica de SAOS (síndrome de apnea obstructiva del sueño), insuficiencia venosa de miembros inferiores, insuficiencia cardiaca, gonartrosis, enfermedad pulmonar obstructiva crónica (EPOC), cardiopatía isquémica (CPI), colelitiasis, AVC/AIT, hiperuricemia, niveles de colesterol total, LDL, HDL y triglicéridos.

El peso se obtuvo con balanzas electrónicas, de lectura digital, con una precisión de +/- 100 gramos, realizándose una calibración para ambas balanzas cada uno de los días del estudio. La medida del peso se hacía con el paciente descalzado y con el pijama de hospitalización. Se realizaron al menos dos pesadas. El peso se registró en kilogramos con dos decimales. Para la medición de la talla, se utilizó una cinta métrica flexible pero inextensible, que se fijaba a la pared de la habitación carente de zócalo. Mediante escuadra de carpintero, se señalaba el punto de altura máxima, con el paciente en bipedestación, realizándose posteriormente la medición. Al igual que con el peso, se hicieron dos mediciones y en caso de no ser coincidentes, se procedía a una tercera. Los resultados se tomaron en centímetros con dos decimales. El indicador ponderal utilizado para valorar el exceso de peso ha sido el IMC, en función de los criterios propuestos por la SEEDO, según el cual se consideran en bajo peso valores menores de $20 \mathrm{Kg} / \mathrm{m}^{2}$, en normopeso los valores entre 20 y $26,9 \mathrm{Kg} / \mathrm{m}^{2}$, en obesidad grado I o sobrepeso los valores comprendidos entre 27 y 29,9 $\mathrm{Kg} / \mathrm{m}^{2}$, en obesidad grado II los valores comprendidos entre 30 y $34,9 \mathrm{Kg} / \mathrm{m}^{2}$, en obesidad tipo III los valores comprendidos entre 35 y $39,9 \mathrm{Kg} / \mathrm{m}^{2}$ y obesidad mórbida o tipo IV valores superiores a $40 \mathrm{Kg} / \mathrm{m}^{2}$. Los pacientes encamados se excluyeron del estudio por la imposibilidad de realizar las medidas pondérales. Para el presenta estudio los grados II, III y IV se han agrupado como una única categoría $\left(\mathrm{IMC} \geq 30 \mathrm{Kg} / \mathrm{m}^{2}\right.$ ) que será referida a partir de ahora con el término obesidad.

El análisis estadístico se ha realizado con el programa SPSS. Se han estimado proporciones con sus intervalos de confianza del 95\%. Para examinar la relación entre la preva- lencia de la obesidad y las variables estudiadas (razón de prevalencia -RP-) se realizaron pruebas de comparación de medias (prueba de la T de Student para variables independientes con análisis de la varianza F de Snedecor) y comparación de proporciones (prueba de la Chi cuadrado). Se admitieron como niveles de significación estadística valores para $\mathrm{p}<0,05$.

\section{RESULTADOS}

La muestra global estudiada fue de 101 sujetos de un total de 128 pacientes (habiéndose excluido 27 por estar encamados). La edad media global fue de 62,14 años, DE 15,7 (hombres $55,4 \%$ y mujeres $44,6 \%$ ). Los hallazgos antropométricos revelaron un peso medio de $71,81 \mathrm{Kg}$, una talla 1.61 metros y un IMC medio $27,41 \mathrm{Kg} / \mathrm{m} 2$ (DE 5,66). La prevalencia de la obesidad estimada para el conjunto de los pacientes ingresados en el Servicio de Medicina interna del Hospital Juan Ramón Jiménez ha sido del 32,7\% [0,236-0,416], siendo del $21,4 \%$ en los hombres y del $46,6 \%$ en las mujeres. La sobrecarga ponderal global (sobrepeso y obesidad o un IMC >27 $\mathrm{Kg} / \mathrm{m} 2$ ) ha sido del $50,5 \%$. El $2 \%$ del colectivo se ha tipificado como obesidad mórbida, el resto de la distribución se representa en la figura 1.

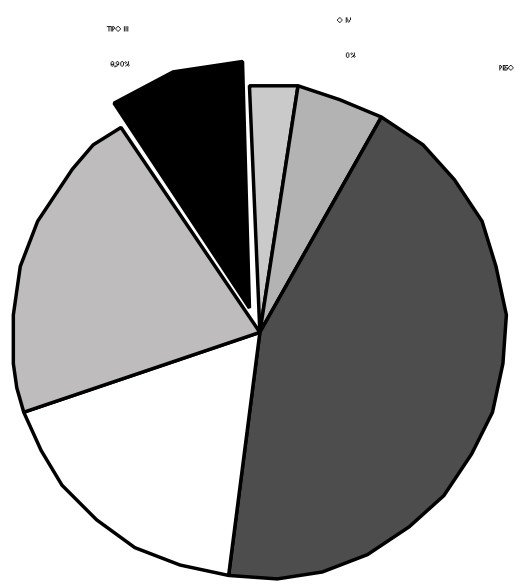

Fig. 1. Distribución de la sobrecarga ponderal según IM C.

En el estudio analítico mediante tablas de contingencia $2 \times 2$, para determinar asociación de prevalencia entre la obesidad y las variables estudiadas, encontramos significación estadística con el género (las mujeres tienen una RP 3,22), la HTA (RP 4,74), la dislipemia (RP 4,40) y la hiperuricemia (RP 4,28). Al límite de la significación quedaron el tabaquismo (RP 0,36), la sospecha clínica de SAOS (RP 5,89) y la gonartrosis (RP 2,59). Los intervalos de confianza y el resto de las variables estudiadas se expresa en la tabla I.

En el estudio por grupos de edades consideramos: < 50 años con una prevalencia de obesidad $15,2 \%, 51-75$ años con una prevalencia de $69,7 \%$ y $<75$ años con $15,2 \%$ (el análisis con x2 no mostró diferencias significativas).

En la comparación de medias de variables cuantitativas sólo los niveles plasmáticos de colesterol total mostró una $\mathrm{p}$ 
TABLA I

DISTRIBUCIÓ N DE LAS VARIABLES ASO CIADAS EN LA DISTRIBUCIÓ N O BESIDAD (IMC > $30 \mathrm{Kg} / \mathrm{M}^{2}$ ), RAZÓ N DE PREVALENCIA (RP) E INTERVALOS DE CO NFIANZA (IC)

\begin{tabular}{lccccc}
\hline & Presencia & Ausencia & RP & IC & P \\
\hline SEXO (M ujer) & $63,6 \%$ & $36,4 \%$ & 3,20 & $1,34-7,62$ & 0,0130 \\
HTA & $75,8 \%$ & $24,2 \%$ & 4,74 & $0,42-2,58$ & 0,00143 \\
DM & $30,3 \%$ & $69,7 \%$ & 1,04 & $0,42-2,58$ & NS \\
DISLIPEM IA & $69,7 \%$ & $30,3 \%$ & 4,40 & $1,79-10,79$ & 0,00179 \\
TABAQUISM O & $24,2 \%$ & $75,8 \%$ & 0,36 & $0,14-0,91$ & 0,047 \\
MENO PAUSIA & $95,2 \%$ & $4,8 \%$ & 5,26 & $0,56-49,28$ & NS \\
IV M M II & $36,4 \%$ & $93,9 \%$ & 1,39 & $0,22-8,79$ & NS \\
ICC & $33,3 \%$ & $63,6 \%$ & 1,85 & $0,75-4,58$ & NS \\
GO NARTRO SIS & $33,3 \%$ & $66,7 \%$ & 2,59 & $0,98-6,83$ & NS \\
EPOC & $9,1 \%$ & $90,9 \%$ & 0,27 & $0,07-1,02$ & NS \\
CPI & $36,4 \%$ & $63,6 \%$ & 1,85 & $0,75-4,58$ & NS \\
CO LELITIASIS & $24,2 \%$ & $75,8 \%$ & 2,09 & $0,72-6,06$ & NS \\
HIPERURICEM IA & $36,4 \%$ & $63,6 \%$ & 4,28 & $1,54-11,92$ & 0,0082 \\
AIT/AVC & $18,2 \%$ & $81,8 \%$ & 1,28 & $0,42-3,91$ & NS \\
SAOS & $15,2 \%$ & $84,8 \%$ & 5,89 & $1,07-32,20$ & NS \\
\hline
\end{tabular}

0,026; el resto de las variables se muestra en la tabla II. En la distribución por géneros, las mujeres presentaron un IMC mayor al de los varones $(29,93$ frente a 25,38; p 0,0001), así como la edad media $(65,37$ frente a 59,55 años; $\mathrm{p}$ NS).

\section{TABLA ॥}

\begin{tabular}{lccc}
\multicolumn{4}{c}{ TABLA II } \\
\multicolumn{4}{c}{ COM PARACIÓ N DE M EDIAS PARA VARIABLES } \\
CUANTITATIVAS & $($ T-STUDENT) \\
\hline & OBESOS & NO OBESOS & $\mathrm{P}$ \\
\hline EDAD & 63,21 & 61,63 & $\mathrm{NS}$ \\
COLESTERO L & 199,93 & 175,56 & 0,026 \\
HDL & 40,13 & 37,46 & $\mathrm{NS}$ \\
LDL & 121,96 & 115,69 & $\mathrm{NS}$ \\
TRIGLICERIDOS & 143,58 & 126,31 & $\mathrm{NS}$ \\
\hline
\end{tabular}

\section{DISCUSIÓN}

En la actualidad, se reconoce que el sobrepeso y la obesidad constituyen un importante problema de salud pública en las sociedades desarrolladas (1). La obesidad no sólo condiciona una mayor morbilidad en numerosas enfermedades, especialmente las del ámbito cardiovascular (9), sino que aumenta la mortalidad total, siendo esta proporcional al incremento del IMC (10). La antropometría, y especialmente el IMC es la herramienta básica para la cuantificación de la grasa corporal estando ampliamente validado por numerosos comités de expertos $(7,8)$. La prevalencia de la obesidad abarca un amplio abanico según distribución geográfica, edad, sexo y nivel socioeconómico entre otras $(11,12)$.

Existen numerosos estudios de prevalencia en población general, siendo el estudio SEEDO'97 el único trabajo global español con una metodología estandarazida, aunque sus fuentes son encuestas nutricionales del País Vasco (2), Comunidad de Madrid (3), Cataluña (4) y Comunidad Valenciana (5). Estiman una prevalencia global $13,4 \%$ (11,5\% en varones y $15,2 \%$ en mujeres) con un aumento progresivo a medida que avanza la edad, alcanzando hasta el 26,3\% en el grupo 55-60 años, especialmente en el subgrupo femenino con menor nivel de instrucción (6).

Que la prevención primaria de la obesidad debería ocupar un lugar destacado para reducir la incidencia de este factor de riesgo, con las importantes repercusiones sociosanitarias y económicas que conlleva es un hecho consumado (11). Sin embargo, aunque el tratamiento de la obesidad es complejo y con alto porcentaje de fracasos, el clínico desde la cabecera del enfermo tiene un papel capital importancia en la detección y control de este factor de comorbimortalidad para sus pacientes. En este sentido, conocer la prevalencia de la obesidad de nuestros enfermos es el primer eslabón para realizar prevención secundaria.

La prevalencia estimada del $32,7 \%$ es superior a la encontrada en otros estudios epidemiológicos realizados sobre población general, pero en nuestro trabajo el objetivo era estudiar enfermos hospitalizados, muchos de ellos con entidades nosológicas graves que han evolucionado de forma insidiosa por su asociación al aumento de grasa corporal. Sin embargo, hemos obtenido resultados similares, demostrando mayor "presencia" de la obesidad entre las mujeres y en el grupo de edad entre 51 y 75 años. En el SEEDO'97 la edad contemplada fue entre 25 y 60 años (6), así pues, estudiamos a una población de mayor edad. Por otra parte, encontramos asociación con los factores de riesgo cardiovasculares clásicos como la hipertensión arterial y la hipercolesterolemia, excluyéndose la Diabetes Mellitus.

La práctica rutinaria del cálculo del IMC es un pilar básico en el conocimiento y manejo clínico de la obesidad, no debiéndose incurrir en datos antropométricos declarados ya que los sujetos tienden a subestimar el peso, y por el contrario sobrees- 
timar la talla con la consiguiente subestimación de este problema de salud, cuestionándose la validez de este método (13).

Además de la morbimortalidad que genera la obesidad, tenemos que comentar la calidad de vida percibida por el paciente. Las medidas de calidad de vida se clasifican generalmente como genéricas y específicas. Las escalas específicas para medir calidad de vida en la obesidad son escasas en la bibliografía, subrayando varios cuestionarios tales como: Impact of Weight on quality of life (IWQOL) (14) y el Obesity related problem (OP) scale utilizado en el estudio Swedish Obese subjects (15). En los resultados obtenidos hasta la

\section{Bibliografía}

1. Björntorp P. Obesity: a chronic disease with alarming prevalence and consequences. J Intern Med 1998; 244: 267-9.

2. Aranceta J, Pérez C, Marzana I, Egileor I, Gonzalez de Galdeano L, Sáez de Buruaga J. Encuesta de nutrición de la comunidad autónoma Vasca. Tendencias de consumo alimentario, indicadores bioquímicos y estado nutricional de la población adulta. Vitoria: Servicio de publicaciones del gobierno vasco, 1994.

3. Aranceta J, Pérez C, Amela C, García Herrera R. Encuesta de nutrición de la comunidad de Madrid. Madrid: consejería de saludo de la comunidad de Madrid, 1994.

4. Serra Majem L, Ribas Barba L, García Closas R, Ramón Torrel JM, Salvador Castell G, Farran Codina A, et al. Avaluació de l'estat nutricional de la població catalana (1992-1993). Avaluació dels habits alimentaris, el consum d'aliments, energia i nutrients, i de l'estat nutricional mitjançant indicadors bioquimics i antropometrics. Barcelona: Generalitat de Catalunya. Departament de sanitat i seguretat social, 1996.

5. Quiles Izquierdo J, Vioque J. Prevalencia de obesidad en la comunidad Valenciana. Med Clin (Barc) 1996; 106: 529-33.

6. Aranceta J, Pérez Rodrigo C., Serra Majem L., Ribas L., Quiles Izquierdo J., Vioque J., et al. Prevalencia de la obesidad en España: estudio SEEDO'97. Med Clin (Barc) 1998; 111: 441-5.

7. WHO-EURO Measuring obesity classification and description of anthropometric data. Report on a WHO consultation on the epidemiology of obesity. Copenhague: WHO-EURO 1987. fecha, los hombres y las mujeres con obesidad grave refieren un peor estado de salud actual, un estado de ánimo menos positivo y una mayor disfunción social, en comparación con la población general (15).

No sólo la prevención primaria de la obesidad debe ocupar un lugar destacado en la lucha para reducir este factor de riesgo, sino que también el clínico tiene que participar en la detección y tratamiento de la sobrecarga ponderal una vez establecida. La elevada prevalencia de la obesidad en población enferma además de aumentar la morbilidad y mortalidad global, condiciona una negativa calidad de vida percibida.

8. Sociedad Española para el Estudio de la Obesidad (SEEDO) Consenso español 1995 para la evaluación de la obesidad y para la realización de estudios epidemiológicos. Med Clin (Barc) 1996; 107: 782-7.

9. Brochu M., Poehlmen, Eric T., Ades Philip A. Obesity, body fat distribution, and coronary artery disease. J Cardiopul Rehabil 2000; 20: 96-108.

10. Stevens J, Cai J, Pamuk ER., Williamson DF., Thun MJ., Wood JL, The effect of age on the association between body-mass index and mortality. N Engl J Med 1998; 338; 1-7.

11. Gutierrez-Fisac JL. La obesidad: una epidemia en curso. Med Clin (Barc) 1998; 111: 456-8.

12. Seidell JC. Time trends in obesity: an epidemiological perspective. Horm Metab Res 1997; 29: 155-8.

13. Quiles Izquierdo J, Vioque J. Validez de los datos antropométricos declarados para la determinación de la prevalencia de obesidad. Med Clin (Barc) 1996; 106: 725-9.

14. Kolotkin RL, Head S, Hamilton M, y cols. Assessing impact of weight on quality of life. Obes Res 1995; 3:49-56.

15. Sullivan M, Karlsson J, Sjostrom L, et al. Swedish obese subjects (SOS): An intervention study of obesity. Baseline evaluation of heakth and psychosocial functioning in the first 1743 subjects examined. Int J Obes Relat Metab Disod 1993; 17: 503-12. 